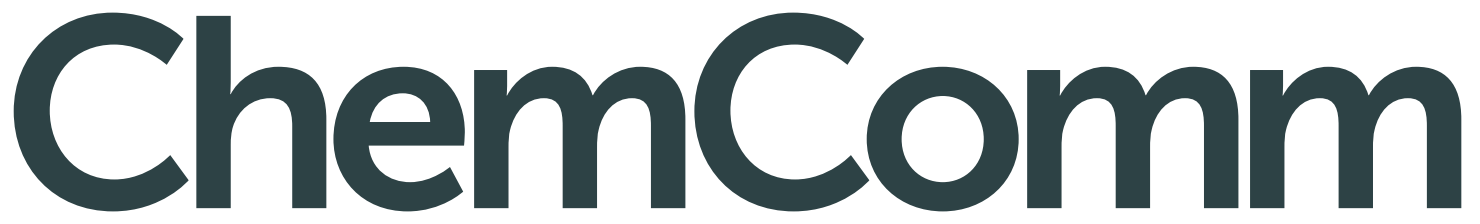

Chemical Communications

rsc.li/chemcomm

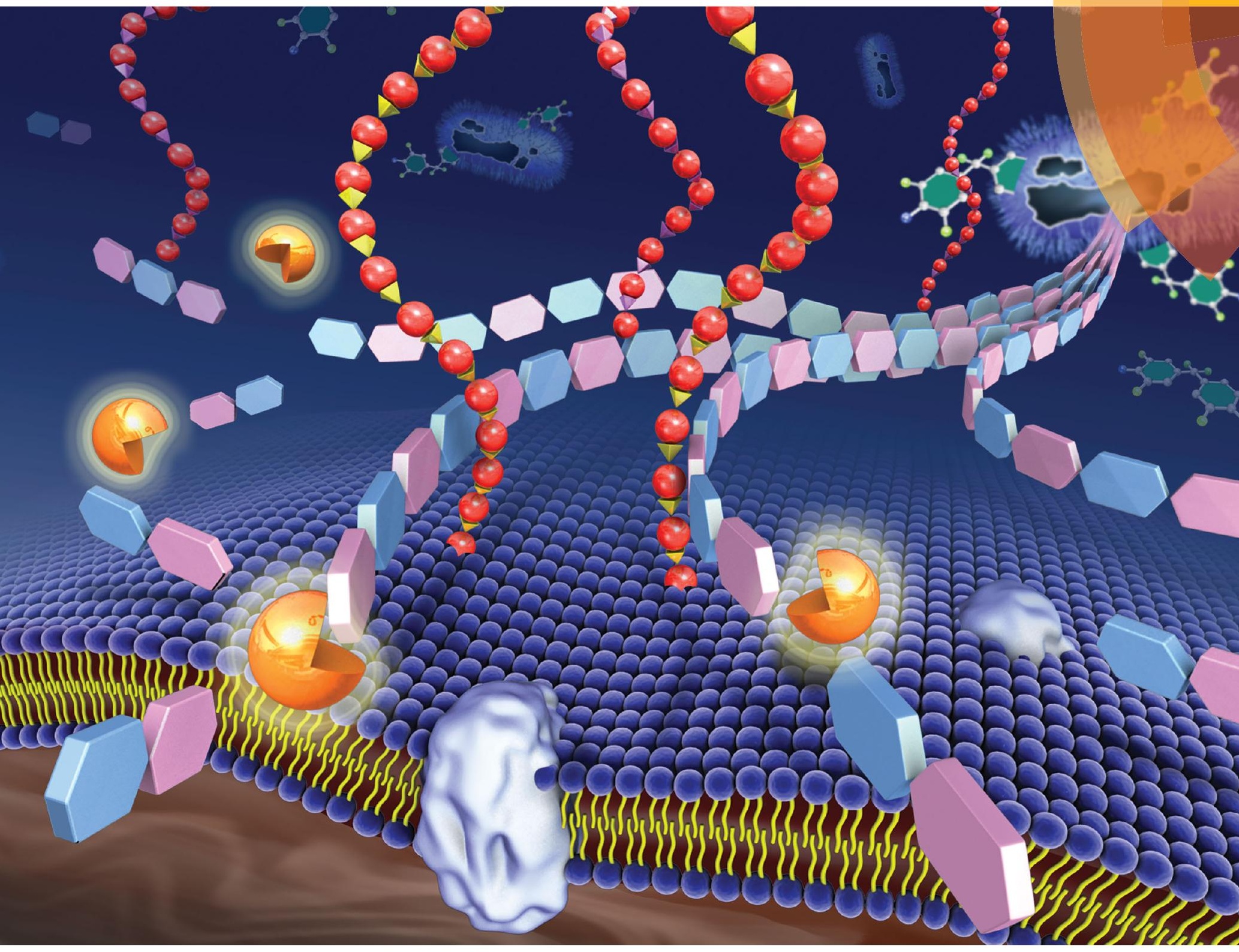

ISSN 1359-7345

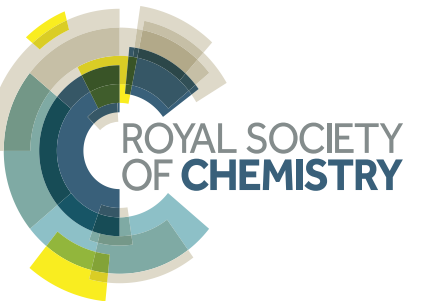




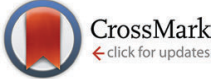

Cite this: Chem. Commun., 2017 53, 3512

Received 23rd January 2017,

Accepted 22nd February 2017

DOI: 10.1039/c7cc00613f

rsc.li/chemcomm

\section{Tetrabromobisphenol A (TBBPA) exhibits specific antimicrobial activity against Gram-positive bacteria without detectable resistance $\dagger$}

\author{
Fang Ji, $\ddagger^{\mathrm{a}}$ Chang Wang $\ddagger^{\mathrm{b}}$ Huimin Wang, $\ddagger^{\mathrm{b}}$ Guangliang Liu, ${ }^{\mathrm{b}}$ Bolei Chen, ${ }^{\mathrm{c}}$ \\ Ligang $\mathrm{Hu}^{d}{ }^{d}$ Guibin Jiang, ${ }^{d}$ Maoyong Song ${ }^{d}$ and Yong Liang*abe
}

We report here the antimicrobial ability of tetrabromobisphenol A (TBBPA) against Gram-positive bacteria without detectable resistance. Microscopy imaging and gene analysis reveal that the bacterial cell wall is the target of TBBPA. Structure-activity studies of TBBPA and its analogues relate the observed antimicrobial activity to the halogen substitution neighboring the phenolic hydroxyl group.

Rapid development of antibacterial resistance remains a challenge for effectively controlling pathogenic microorganisms. ${ }^{1}$ Continuous efforts have been made to battle antibacterial resistance through exploring the potential options of using natural products and synthetic compounds of antimicrobial activity. ${ }^{2}$ Tetrabromobisphenol A (TBBPA) is a synthetic, highly lipophilic halogenated aromatic molecule, and has been widely used as a brominated flame retardant to reduce the risk of ignition and to prevent firerelated damage. ${ }^{3}$ In our work of investigating the toxicological effects of TBBPA on soil microorganisms, we found that TBBPA can specifically inhibit the growth of a range of Gram-positive bacteria, but has no effect on the tested Gram-negative bacteria. Literature search found that TBBPA has not been reported for its antibacterial activity (to our best knowledge), but a report published in Japanese in 1952 suggested the antifungal activity of chlorinated bisphenols. ${ }^{4}$ The observation of TBBPA inhibiting Gram-positive bacteria and the previously reported antifungal activity of its chlorinated analogs prompted us to reason that

\footnotetext{
${ }^{a}$ School of Medicine, Jianghan University, Wuhan 430056, P. R. China. E-mail: ly76@263.net

${ }^{b}$ Institute of Environment and Health, Jianghan University, Wuhan 430056, P. R. China

${ }^{c}$ Institute for Interdisciplinary Research, Jianghan University, Wuhan 430056, P. R. China

${ }^{d}$ State Key Laboratory of Environmental Chemistry and Ecotoxicology, Research Center for Eco-Environmental Sciences, Chinese Academy of Sciences, P.O. Box 2871, Beijing 100085, P. R. China

${ }^{e}$ Key Laboratory of Optoelectronic Chemical Materials and Devices

(Jianghan University), Ministry of Education, Wuhan 430056, P. R. China

$\dagger$ Electronic supplementary information (ESI) available. See DOI: 10.1039/ c7cc00613f

$\ddagger$ Equal contribution.
}

TBBPA may potentially have promising antimicrobial effects. Therefore, we then conducted studies focusing on its antibacterial activity, and report herein the discovery of the specific antimicrobial activity of TBBPA toward Gram-positive bacteria without detectable resistance.

In the susceptibility studies, typical Gram-positive bacteria showed susceptibility to TBBPA. The minimum inhibition concentrations (MIC) of TBBPA for the tested representative bacteria ranged from 2 to $8 \mu \mathrm{g} \mathrm{mL}{ }^{-1}$ in the order Staphylococcus aureus $<$ Bacillus subtilis $=$ Bacillus thuringiensis $<$ Streptomyces coelicolor (Table 1).

Even the methicillin resistant-Staphylococcus aureus (MRSA) could be inhibited by low levels of TBBPA. In contrast, no inhibition effect was observed for the typical Gram-negative bacteria, e.g., Escherichia coli, Agrobacterium tumefaciens, Xanthomonas oryzae, Pseudomonas syringae and Ralstonia solanacearum, as the MICs were no less than $128 \mu \mathrm{g} \mathrm{mL}{ }^{-1.5}$. We tried to evaluate the antimicrobial resistance of Bacillus subtilis strain 168 (Bs168) to TBBPA by stepwise growing and exposing it in increasing concentrations $(1 / 2 \times \mathrm{MIC})$, but failed to produce TBBPA-resistant mutants of Bs168 even when the concentration of TBBPA was up to 4 fold of MIC (Fig. 1). In fact, Bs168 could not continuously grow in such high concentrations of TBBPA (relative to the MIC) and therefore we were unable to culture the

Table 1 Minimal inhibition concentrations (MICs) of TBBPA on the tested bacteria

\begin{tabular}{lr}
\hline Strain & MIC $(\mu$ \\
\hline Gram-positive bacteria & \\
Bacillus subtilis strain 168 & 4 \\
Staphylococcus aureus 25923 & 2 \\
MR - Staphylococcus aureus & 4 \\
Bacillus thuringiensis ssp. finitimus 020 & 4 \\
Streptomyces coelicolor M145 & 8 \\
& \\
Gram-negative bacteria & \\
Escherichia coli DH5a & 128 \\
Agrobacterium tumefaciens GV3101 & $>128$ \\
Pseudomonas syringae pv. tomato DC3000 & $>128$ \\
Xanthomonas oryzae pv. oryzae & $>128$ \\
Ralstonia solanacearum SD1 & $>128$
\end{tabular}




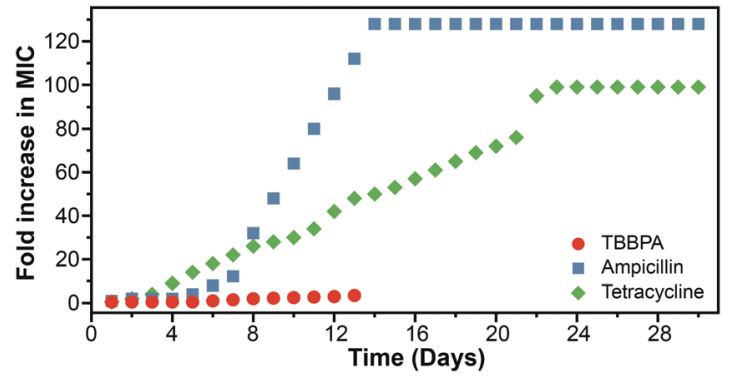

Fig. 1 Antimicrobial resistant characterization of Bs168 to TBBPA, ampicillin, and tetracycline. With increasing concentration (up to 4-fold MIC), TBBPA inhibited the growth of Bs168 and the bacteria could not be cultured after 13 days (and thus no data were present after 13 days for TBBPA incubation).

bacteria after around 13 days, as shown by the lack of data after 13 days for TBBPA exposure in Fig. 1. However, we obtained the tetracycline- and ampicillin-resistant strains of Bs168 by the same screening methods (Fig. 1).

The non-detectable resistance of TBBPA antibiotic effect further prompts the potential of TBBPA to be used as an antimicrobial agent. Subsequently, we used Bs168, a typical model of Grampositive bacteria, ${ }^{6}$ to conduct a series of experiments to characterize the antimicrobial activity of TBBPA.

We studied cell morphology using scanning and transmission electron microscopy (SEM and TEM), after adding TBBPA (at a final concentration of $16 \mu \mathrm{g} \mathrm{mL}{ }^{-1}$, DMSO treatment as control) to the culture of Bs168 with an $\mathrm{OD}_{600}$ of approximately 0.6-0.8. It was observed that the Bs168 cells, which were harvested $1 \mathrm{~h}$ later, lost the normal cell morphology of a rod shaped cylinder (Fig. 2A) and were severely torn open upon TBBPA exposure (Fig. 2B). The TEM micrographs showed that, while the control cells had an intact cell envelope with a substantial amount of dark dense content inside (Fig. 2C), the TBBPA-treated cells had small crevices and large holes in the envelope with little dark content inside (Fig. 2D and E).

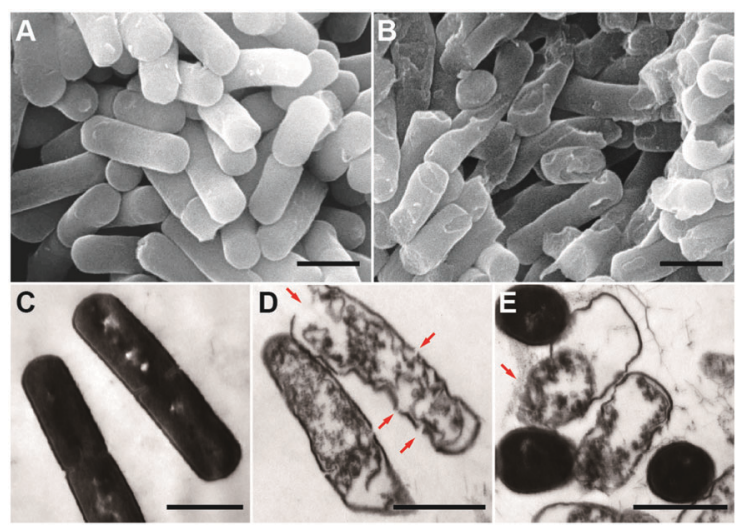

Fig. 2 TEM and SEM images of TBBPA-exposed Bs168. The culture of Bs168 with an $\mathrm{OD}_{600}$ of approximately $0.6-0.8$ was treated with $16 \mu \mathrm{g} \mathrm{mL}^{-1}$ of TBBPA ( $B, D$ and $E$ ) or an equivalent amount of $D M S O$ as control ( $A$ and $C$ ) for $1 \mathrm{~h}$. The treated cells were studied using SEM (A and B) and TEM (C-E). Arrows indicate the small crevices on the cell wall. (B) A severely damaged cell wall and (E) the protoplast leaking out. The scale bars are $1 \mu \mathrm{m}$ for panels $(A-E)$.
Table 2 Comparisons in the chemical structure and MICs for Bs168 between TBBPA and its analogous compounds

\begin{tabular}{|c|c|c|c|}
\hline Name & Structure & $\operatorname{MIC}\left(\mu \mathrm{g} \mathrm{mL} L^{-1}\right)$ & $\operatorname{MIC}_{\mathrm{m}}\left(10^{-6} \mathrm{~mol} \mathrm{~L}^{-1}\right)$ \\
\hline TBBPA & & 4 & 7.3 \\
\hline TCBPA & & 4 & 10.9 \\
\hline BPA & & 128 & 560.7 \\
\hline TBBPADE & & $>128$ & $>205.1$ \\
\hline TBBPABE & & $>128$ & $>202.5$ \\
\hline TBBPS & & $>128$ & $>226.2$ \\
\hline
\end{tabular}

It appeared that most of the intrinsic content inside the TBBPAtreated cells flew out through those crevices and holes or was degraded by enzymes inside the dying cells. The TBBPA-treated cells broke up, probably due to these damages to the cell wall (Fig. 2E).

In order to explore the structural features responsible for the antimicrobial activity, we examined the antimicrobial activity of five compounds structurally similar to TBBPA. The MIC values expressed as molarity $\left(\mathrm{MIC}_{\mathrm{m}}\right)$ were also calculated for comparison (Table 2). It was observed that only tetrachlorobisphenol A (TCBPA), an analog of TBBPA with $\mathrm{Cl}$ replacing $\mathrm{Br}$, showed antibacterial activity against Bs168 with the same MIC of $4 \mu \mathrm{g} \mathrm{mL}{ }^{-1}$, with the others showing no considerable antibacterial effect (MICs $>$ $128 \mu \mathrm{g} \mathrm{mL}{ }^{-1}$ ) (Table 2). It seemed that the structural features of both halogen substitution neighboring the phenolic hydroxyl groups and the bridge linking two aryl rings played a key role in the antimicrobial activity of BPA analogues. Brominated BPA exhibited a stronger antimicrobial activity than the chlorinated analog, while halogenated substituents on the phenyl rings produced $\mathrm{MIC}_{\mathrm{m}}$ of BPA over 50 times higher than those of TCBPA and TBBPA. Moreover, upon calculating the relative antimicrobial activity (RAA) expressed in terms of percentage with $100 \%$ being defined as the lowest $\mathrm{MIC}_{\mathrm{m}}$ value of $7.3 \times 10^{-6} \mathrm{~mol} \mathrm{~L}^{-1}$, which was observed for TBBPA, the relative activity of TCBPA was found to be $67.3 \%$, considerably lower than that of TBBPA. BPA exhibited a much weaker relative activity of $1.3 \%$.

We further attempted to identify the causes of the antibacterial activity of TBBPA against Gram-positive bacteria by investigating the global transcription profiles using microarray chips of Bs168 following exposure to TBBPA. ${ }^{7}$ The complete genome of Bs168 was sequenced in 1997 and reported to encode 4106 proteins. ${ }^{8}$ In our study, differentially expressed gene analysis showed that, after $60 \mathrm{~min}$ of TBBPA treatment, 763 genes were significantly $(p<0.05)$ changed with a fold change of $>2$ compared to controls, with 449 genes being up-regulated and 314 down-regulated, including a considerable number of genes of unknown function (Fig. 3).

Considering that TBBPA specifically acts against Grampositive bacteria and probably targets the cell wall, we focused gene analysis on the differences in cell walls between Grampositive and -negative bacteria. A significant known difference 


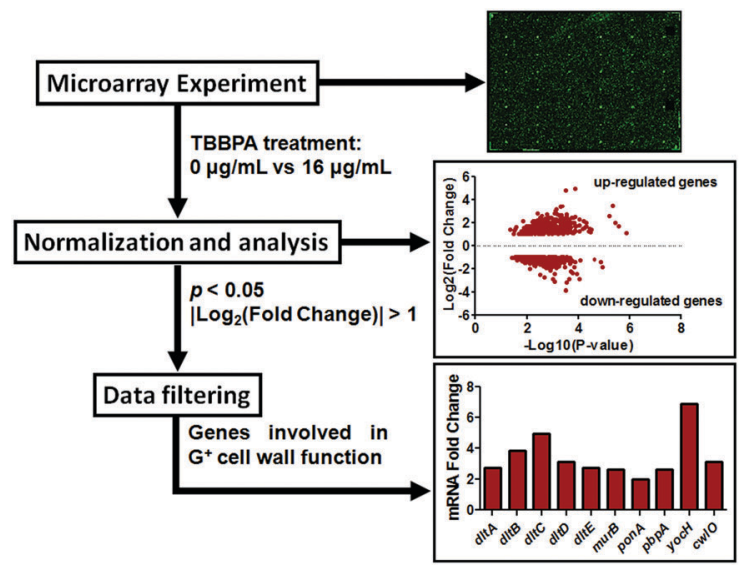

Fig. 3 Results of microarray analysis of mRNA expression in control and TBBPA treated Bs168. Volcano plot analysis applied to the microarray data revealed that 763 genes were significantly $(p<0.05)$ changed with a fold change of $>2$ (up or down) in TBBPA treated group compared to controls. The plot shows the negative log of $t$-test $p$-values between two groups on the $X$-axis and the $\log 2$ (fold change) in mRNA expression on the $Y$-axis. Each gene was represented by a single point. Based on the specificity of TBBPA antibacterial activity on Gram-positive bacteria and the differences in genes between Gram-positive and Gram-negative bacteria, 10 significantly changed genes that are specific to Gram-positive bacteria were selected and enriched, reasoning that they are involved in the cell wall function of Gram-positive bacteria which is the specific target of TBBPA.

between these two types of bacteria is the presence of teichoic acids (TAs), specifically in Gram-positive bacteria cell walls. As TAs are negatively charged, D-alanine is protonated and covalently linked to the TAs providing counter ions determining the net negative charge of the TAs and maintaining the intactness and functions of the cell wall. ${ }^{10}$ The addition of D-alanyl to TAs is regulated by the dlt operon, as DltA ligates D-alanine in the cytoplasm of the cell and the ligated form is then transported by the carrier protein DltC. ${ }^{9}$ The negatively charged D-alanyl-TAs provide binding sites for the cationic autolysins, which regulates cell autolysis, ${ }^{11}$ playing an important role in maintaining the dynamic structure of the bacterial cell wall through coordinated synthesis and degradation. ${ }^{12}$

Based on the aforementioned specificity of the cell wall structure in Gram-positive bacteria, we focused our gene analysis on the Dlt family and autolysin genes. We indeed observed that a gene cluster of dltA, B, C, D and $E$ (BSU38500, BSU38510, BSU385200, BSU38530 and BSU38540) was induced by more than 3-fold, while two autolysin genes yocH and $c w l O$ (BSU19210 and BSU34800) were up regulated by more than 6 and 3-fold, respectively (Fig. 3). It seems that the up-regulation of $d l t$ and autolysin genes could be a major factor that (at least in large part) caused cell wall damage. In addition, we performed qRT-PCR assay to verify the transcription change of these two genes. Similar results proved that autolysins might be involved in the cell wall damage (Fig. S1, ESI $\dagger$ ).

To further verify the involvement of Dlt genes in the effects of TBBPA damaging the cell wall and showing the antibacterial activity, the transcriptional changes of the dltA gene of Bs168 upon exposure to TBBPA and its analogs were investigated. From the antibacterial activity results shown in Table 2 , it is seen that only TBBPA and TCBPA dose-dependently up-regulated dltA, but

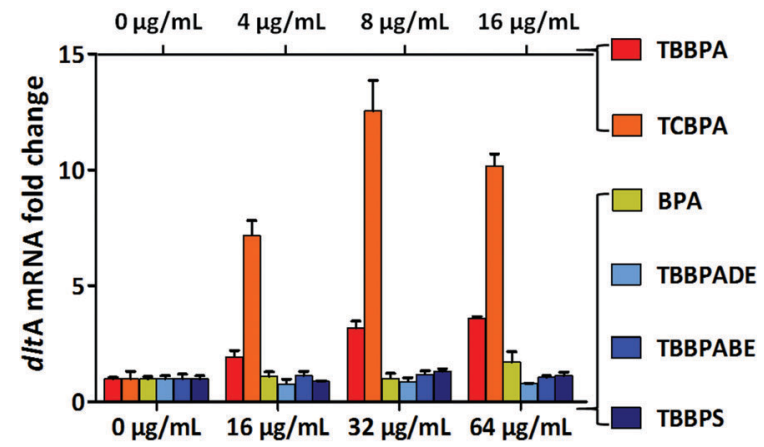

Fig. 4 Effects of TBBPA, TCBPA, BPA, TBBPADE, TBBPABE and TBBPS on dltA mRNA. Bs168 was grown to an $O_{600}$ of 0.6-0.8 and incubated for 60 min with the addition of different concentrations of each compound. qRT-PCR was performed to measure the levels of $d l t A$ mRNA with primers specific for $d l t A$ and for rpsJ gene as the internal control. Mean values from three different experiments are shown. The error bars indicate \pm standard deviations. The results shown are representative of experiments performed at least three times.

not the other compounds (Fig. 4). These results provided additional support to the notion that the antibacterial activity of TBBPA may be related to the up-regulation of $d l t$ and autolysin genes.

Based on these results, we gave the following explanations about the antibacterial activity of TBBPA through up-regulating Dlt and autolysin genes causing cell wall damages of Gram-positive bacteria. Upon the exposure of Bs168 cells to TBBPA, TBBPA may interact with the Dlt protein, which subsequently results in the interruption of D-alanine modification and insertion into TAs. This would have two consequences. First, as a feedback to the loss of the Dlt enzyme function, cells would up-regulate dlt genes, as evidenced by our gene analysis results. Second, as less D-alanine was ligated and integrated with TAs, the cell wall would have more negative charge and thus have more binding sites for autolysins, resulting in increased activities of autolysins. Consequently, the increased activities of autolysins led to cell lysis, which echoed the expression enhancement of two autolysin genes, yocH and $\mathrm{cwlO}$, which are responsible for encoding D,L-endopeptidases and amidase. ${ }^{13}$ We observed that yocH and $c w l O$ were up regulated by more than 6 and 3-fold, respectively (Fig. 3). Our results agree well with previous reports that the removal of the D-alanyl esters could induce cell lysis, ${ }^{12 c, 14}$ suggesting that our explanations on the causes of TBBPA exposure damaging the cell wall of Grampositive bacteria are reasonable. A schematic diagram describes the possible process of cell wall damage upon TBBPA exposure (Fig. S2, ESI $\dagger$ ).

Although our studies here tend to suggest that the up-regulation of $d l t$ genes and autolysin genes is the possible primary cause of cell lysis, other pathways might still be possible. For instance, in the gene-expression experiment, we observed that 3 peptidoglycansynthesis related genes, including murB (BSU15230) and two penicillin-binding proteins (PBPs) encoding genes, ponA and pbpA (BSU22320 and BSU25000), were induced by over 2-fold (Fig. 3). The over-expression of these genes might also be related to the antimicrobial activity of TBBPA, and a more detailed examination of gene responses is needed to fully characterize the antimicrobial action of TBBPA. 
Nonetheless, we found in this study that TBBPA has high antibacterial activity specifically against Gram-positive bacteria without antibiotic resistance. Considering that TBBPA is an easily synthesized low-molecular weight compound with good stability, TBBPA appears to be a promising candidate as a novel antimicrobial agent against Gram-positive bacteria. For instance, it seems promising to embed TBBPA into certain types of membranes for controlling infections caused by Gram-positive bacteria (e.g., Staphylococcus aureus) arising in association with wounds and surgical operations, although further studies are warranted for these purposes.

This work was financially supported by grants from the Strategic Priority Research Program of the Chinese Academy of Sciences (XDB14030501), the National Nature Science Foundation of China $(21277062,21477049,21507155)$ and the State Key Laboratory of Environmental Chemistry and Ecotoxicology, RCEES, CAS (KF2011-06).

\section{Notes and references}

1 P. Wright, L. Seiple and A. Myers, Angew. Chem., Int. Ed., 2014, 53, 8840. 2 L. Ling, et al., Nature, 2015, 517, 455; M. Yin, Z. Li, E. Ju, Z. Wang, K. Dong, J. Ren and X. Qu, Chem. Commun., 2014, 50, 10488;
J. Hoque, M. Konai, S. Samaddar, S. Gonuguntala, G. Manjunath, C. Ghosh and J. Haldar, Chem. Commun., 2015, 51, 13670.

3 O. Segev, A. Kushmaro and A. Brenner, Int. J. Environ. Res. Public Health, 2009, 6, 478.

4 K. Okazaki, T. Kawaguchi and K. Matsui, Yakugaku Zasshi, 1952, $72,1403$.

5 R. J. Heath, J. Li, G. E. Roland and C. O. Rock, J. Biol. Chem., 2000, 275, 4654.

6 T. J. Silhavy, D. Kahne and S. Walker, CSH Perspect. Biol., 2010, 2, 16.

7 M. D. Brazas and R. E. W. Hancock, Drug Discovery Today, 2005, 10, 1245; M. Cao, T. Wang, R. Ye and J. D. Helmann, Mol. Microbiol., 2002, 45, 1267; E. B. Goh, G. Yim, W. Tsui, J. McClure, M. G. Surette and J. Davies, Proc. Natl. Acad. Sci. U. S. A., 2002, 99, 17025.

8 F. Kunst, et al., Nature, 1997, 390, 249.

9 N. T. Reichmann, C. P. Cassona and A. Grundling, Microbiology, 2013, 159, 1868.

10 H. Yonus, P. Neumann, S. Zimmermann, J. J. May, M. A. Marahiel and M. T. Stubbs, J. Biol. Chem., 2008, 283, 32484.

11 S. P. Falk, J. W. Noah and B. Weisblum, Antimicrob. Agents Chemother., 2010, 54, 3723.

12 (a) F. C. Neuhaus and J. Baddiley, Microbiol. Mol. Biol. Rev., 2003, 67, 686; (b) A. Steen, E. Palumbo, M. Deghorain, P. S. Cocconcelli, J. Delcour, O. P. Kuipers, J. Kok, G. Buist and P. Hols, J. Bacteriol., 2005, 187, 114; (c) J. Wecke, M. Perego and W. Fischer, Microb. Drug Resist., 1996, 2, 123.

13 I. M. Shah and J. Dworkin, Mol. Microbiol., 2010, 75, 1232; D. Noone, L. I. Salzberg, E. Botella, K. Basell, D. Becher, H. Antelmann and K. M. Devine, J. Bacteriol., 2014, 196, 237.

14 W. Fischer, P. Rosel and H. U. Koch, J. Bacteriol., 1981, 146, 467. 